\title{
A!
}

This is an electronic reprint of the original article.

This reprint may differ from the original in pagination and typographic detail.

Asrese, Alemnew; Walelgne, Ermias; Lutu, Andra; Alay, Ozgu; Ott, Jörg

\section{Measuring Web Latency in Cellular Networks}

DOI:

10.1109/INFCOMW.2018.8406855

Published: 01/01/2018

Document Version

Peer reviewed version

Please cite the original version:

Asrese, A., Walelgne, E., Lutu, A., Alay, O., \& Ott, J. (2018). Measuring Web Latency in Cellular Networks. Abstract from IEEE Conference on Computer Communications, Honolulu, Hawaii, United States.

https://doi.org/10.1109/INFCOMW.2018.8406855

This material is protected by copyright and other intellectual property rights, and duplication or sale of all or part of any of the repository collections is not permitted, except that material may be duplicated by you for your research use or educational purposes in electronic or print form. You must obtain permission for any other use. Electronic or print copies may not be offered, whether for sale or otherwise to anyone who is not an authorised user. 


\title{
Poster Abstract: Measuring Web Latency in Cellular Networks
}

\author{
Alemnew Sheferaw Asrese*, Ermias Andargie Walelgne*, Andrua Lutu ${ }^{\dagger}$, Özgu Alay ${ }^{\dagger}$ and Jörg Ott ${ }^{\ddagger}$ \\ ${ }^{*}$ Aalto University, Finland ${ }^{\dagger}$ Simula Research Laboratory ${ }^{\ddagger}$ TU Munich, Germany
}

\begin{abstract}
This work presents a new methodology to measure the performance of web browsing over operational Mobile Broadband (MBB) networks. We designed a web performance measurement tool that collects both the web QoS metrics and the web rendering time in a browser window. We used MONROE [1], European wide measurement platform, to deploy our tool and to conduct a web browsing measurement over operational MBB networks. The results from the initial deployment show that different operators across countries and within the same country have a significant difference in web browsing performance (e.g. in the median case TIM is $\mathbf{7 5}$ ms faster than I WIND regarding time to first byte (TTFB)).
\end{abstract}

\section{INTRODUCTION}

The fast adoption of mobile devices has changed the way we the people want to interact and access to social media, e-commerce, entertainment applications, etc. Recent studies show that mobile data traffic is increasing exponentially, and web browsing is among the dominant applications on a cellular network [3]. Thus, for cellular network operators it is crucial to understand the quality of experience (QoE) that their end customers get. We believe that mobile users care more about the web QoE rather than the quality of service metrics like throughput.

There have been many open measurement tools and platforms that aim to measure the performance of MBB network. NetRadar [6], SamKnows broadband measurement [5], are among these tools that have been developed and deployed on Smartphones to measure the Quality of Service (QoS) metrics. These tools measure not only the metrics which are related with the network QoS such as latency, packet loss, download and upload speed but also the metrics related with the cellular network information like received signal strength, Cell ID, location, etc.

Meteor [4] is another measurement tool which measures the speed of the network and tells the experience that the user can expect while using selected popular applications given their connection requirements. The methodology used by Meteor is not open and we do not know how the expected experience is computed and which performance metrics are used for a given application. Unlike Meteor we measure different metrics at the network and application level, e.g. page download time, and the rendering time at the browser which more relevant from the user perspective. Due to this, we lack to know the performance of web browsing and the perceived quality of operational networks.

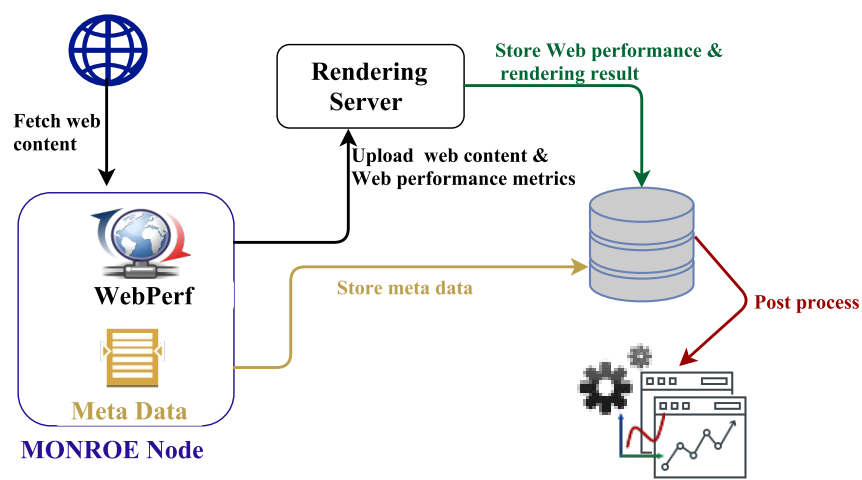

Fig. 1. Measurement Setup

In this work, we present a new approach and a large scale measurement tool for web browsing performance on MBB networks and investigate the impact of network conditions on the end-user experience. Our approach enables us to captured network and web latency metrics such as DNS resolution time and TCP connect time, web complexity metrics like the number of objects, and the page download time, the rendering time and device and network level metadata including the received signal strength and GPS location.

\section{EXPERIMENT DESIGN}

We extended our measurement tool - WePR [2], to capture the web performance and end-user browsing experience in MBB networks. The tool measures the web communication latency metrics such as DNS lookup time, TCP connection time, HTTP download time, and the web complexity metrics including number of HTTP(S) elements, the size and type of the object. Moreover, the tool fetches all the objects of the website at the nodes and pushes them with the recorded metrics to the rendering server so that the web page will be recreated and rendered. Furthermore, we record metadata about the network conditions at the measurement nodes (e.g., MBB coverage profiles, signal strength) and other information that describes the user's condition (e.g. GPS location).

The experimental setup is depicted in Figure 1. The MONROE nodes are embedded devices with cellular network connectivity which enables to run measurement software. We deployed our Web Performance measurement tool in these nodes. The nodes run a service that publish metadata information about the network and device conditions so that an experiment 
that runs in the node can subscribe to the service and get the changes in the network or the device. We record these meta data from the beginning to the end of the experiment. Before starting the execution of the web performance measurement test we capture all the network and device states, and while the experiment is running we record any network or device level state changes or event occurrence (e.g. signal strength, cell ID, GPS location).

The websites are replayed again (based on the metrics recorded and web contents fetched at the MONROE nodes) at the rendering server so as to compute the web rendering time in an actual browser window. The approach of computing the rendering time is described in detail at [2].

\section{RESULTS FROM INITIAL TRIALS}

To perform the web performance measurement in an operational MBB networks, we have been using MONROE [1] measurement platform. The platform comprises both stationary and mobile nodes that are distributed across Italy, Norway, Spain and Sweden. The MONROE measurement nodes are powerful devices that run Linux distribution and connect to a few cellular network providers. The mobile nodes are deployed in trucks, long distance trains, vehicles while the stationary nodes are placed at offices, and homes.

We have been running the experiment in some experimental stationary nodes that are distributed in the four countries mentioned above, and we measured the performance of three most popular websites (www.bbc.com, www.ebay.com, and www.go.com) over cellular networks. From each measurement vantage points, we triggered web browsing to these websites in every hour. We collected about $3 \mathrm{~K}$ data points in August 2016 from 19 measurement nodes

\section{A. Web Performance}

We wanted to see the behaviors of four key web latency metrics (DNS resolution time, TCP connect time, TTFB and the Page Load Time (PLT)) in cellular networks. By using these key web browsing performance metrics, we are more interested to see the performance of web browsing in different operators in the same country and across different countries.

Figure 2, shows the median DNS resolution and TCP connect time over different operators. As it can be seen from the result, operators in the same country have a very big difference in the time taken to resolve a domain name and to establish TCP connection. For instance, in $80 \%$ of the cases the Italian operator, 'I WIND' has the worst DNS resolution performance. Instead the other Italian operator 'TIM' has the best performance in terms of DNS resolution time. For example, 'Telia' which is a Swedish operator has a very good performance in terms TCP connection time (in $99 \%$ of the cases, it finishes within $60 \mathrm{~ms}$ ), and ' $3 \mathrm{SE}$ ', an operator from Sweden has a poor performance (in $80 \%$ of the cases, it takes more than $60 \mathrm{~ms}$ to make a TCP connection to the websites).

We also observe the TTFB and the PLT of the requested webpages over different operators. As shown in Figure 3 there is a significant difference in terms of TTFB and PLT both

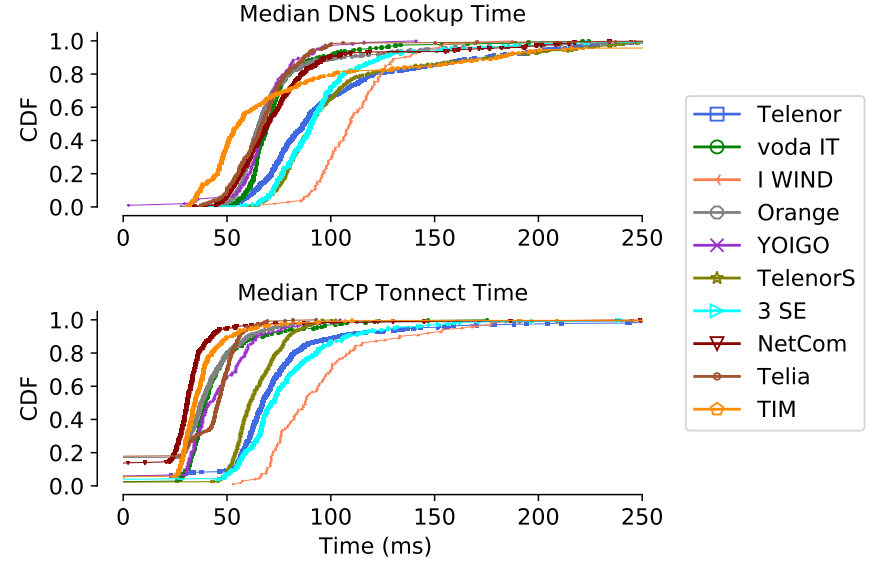

Fig. 2. The DNS resolution and TCP connect time of the three websites over different operators.

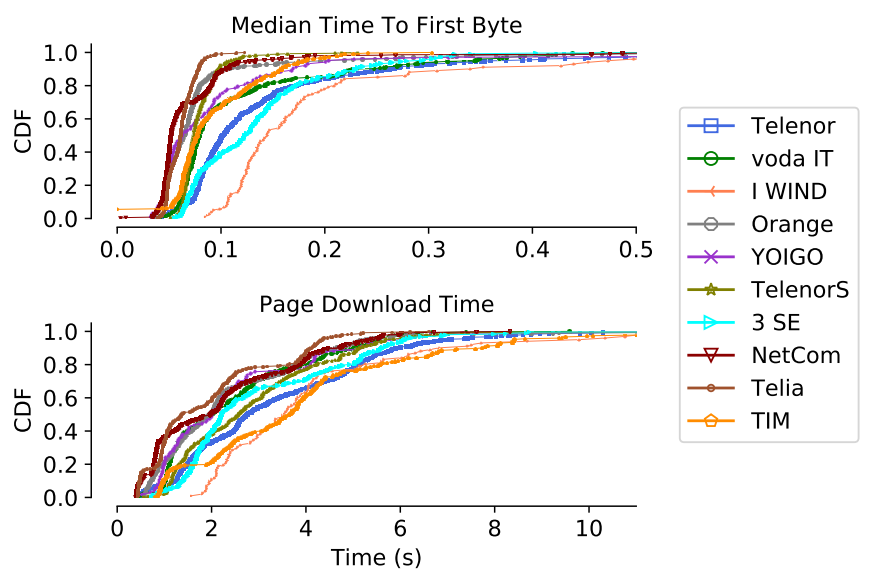

Fig. 3. The time to first byte and the download time of the three websites over different operators.

across a country or within the same country. In fact, the TTFB, TCP connect time and PLT may not be influenced only by the network performance of the operators. The websites may have been served by a CDN which might be located very close to the networks of the operators that show a good performance. One interesting observation is that, operators which have a good performance in terms of the DNS resolution time, TCP connection time, and TTFB may have a poor performance in terms of PLT. For instance, the operator 'TIM' which has a good performance in all the three metrics has almost the same performance with 'I WIND', which has the worst performance in all these web browsing metrics.

\section{REFERENCES}

[1] Ö. Alay, et al. Experience: An Open Platform for Experimentation with Commercial Mobile Broadband Networks. In ACM MobiCom 2017.

[2] A. S. Asrese, et al. WePR: A Tool for Automated Web Performance Measurement. In IEEE GLOBECOMM, QoEMC Workshop 2016.

[3] A. Balachandran, et al. Modeling Web Quality-of-experience on Cellular Networks. In ACM MobiCom 2014.

[4] OpenSignal. Meteor. https://meteor.opensignal.com.

[5] SamKnows. SamKnows. https://samknows.com.

[6] S. Sonntag, et al. Netradar - Measuring the Wireless World. In IEEE WiOpt 2013. 